\title{
Bio-Based Lignin Nanocarriers Loaded with Fungicides as a Versatile Platform for Drug Delivery in Plants
}

Thiago O. Machado, Sebastian J. Beckers, Jochen Fischer, Beate Müller, Claudia Sayer, Pedro H. H. de Araújo, Katharina Landfester,* and Frederik R. Wurm*

Cite This: Biomacromolecules 2020, 21, 2755-2763

Read Online

\section{ACCESS | Lill Metrics \& More | 回 Article Recommendations | sl Supporting Information}

ABSTRACT: Lignin-based nano- and microcarriers are a promising biodegradable drug delivery platform inside of plants. Many wood-decaying fungi are capable of degrading the wood component lignin by segregated lignases. These fungi are responsible for severe financial damage in agriculture, and many of these plant diseases cannot be treated today. However, enzymatic degradation is also an attractive handle to achieve a controlled release of drugs from artificial lignin vehicles. Herein, chemically cross-linked lignin nanocarriers (NCs) were prepared by aza-Michael addition in miniemulsion, followed by solvent evaporation. The cross-linking of lignin was achieved with the bio-

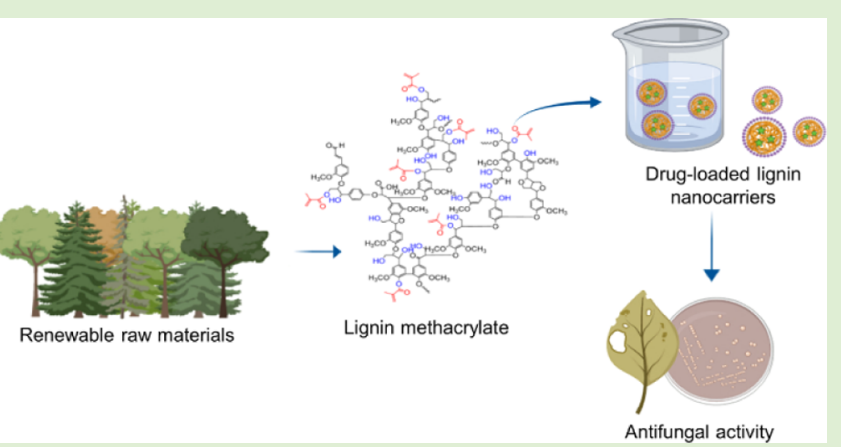
based amines (spermine and spermidine). Several fungicidesnamely, azoxystrobin, pyraclostrobin, tebuconazole, and boscalid-were encapsulated in situ during the miniemulsion polymerization, demonstrating the versatility of the method. Lignin NCs with diameters of 200-300 nm (determined by dynamic light scattering) were obtained, with high encapsulation efficiencies (70-99\%, depending on the drug solubility). Lignin NCs successfully inhibited the growth of Phaeomoniella chlamydospora and Phaeoacremonium minimum, which are lignase-producing fungi associated with the worldwide occurring fungal grapevine trunk disease Esca. In planta studies proved their efficiency for at least 4 years after a single injection into Vitis vinifera ("Portugieser") plants on a test vineyard in Germany. The lignin NCs are of high interest as biodegradable delivery vehicles to be applied by trunk injection against the devastating fungal disease Esca but might also be promising against other fungal plant diseases.

\section{INTRODUCTION}

Lignin has been recently investigated as a renewable, abundant, and inexpensive feedstock to develop sophisticated nanostructures. ${ }^{1-3}$ Lignin is a highly cross-linked aromatic polyetherpolyol that is separated from cellulose and hemicellulose during the pulping process in paper production. Every year several million tons are generated as waste from the Kraft wood pulping under alkaline conditions in combination with sodium sulfide. The majority of this "Kraft lignin" is mainly burned as fuel or is discarded as waste. ${ }^{4,5}$ However, this Kraft lignin is a soluble and multifunctional macromolecule that can undergo chemical modification and thus giving rise to a vast variety of derivatives and fine chemicals. ${ }^{4-9}$ Lignin-derived compounds are useful as biodegradable building blocks for nanomaterials, ${ }^{10}$ and during the past few years, some studies presented lignin-based nanocarriers (NCs). ${ }^{3,11-14}$ Particularly, the development of bio-based and biodegradable nano- and micro-carriers for agriculture was a focus of our recent studies. $^{2,14}$

Nanotechnology in agriculture has become a promising platform for more sustainable release of actives, for example, as nanofertilizers ${ }^{15}$ and nanopesticides ${ }^{16}$ and for genetic engineer- ing. ${ }^{17}$ Nanostructures for agricultural applications ${ }^{18}$ are mainly dispersions, ${ }^{19}$ quantum dots, and metal oxide nanoparticles, and NCs composed of silica, lipids, or polymers. They can be applied to the $\operatorname{soil}^{15}$ or into the plant; ${ }^{2,20}$ upon foliar application, some NCs are able to penetrate the leaves and are translocated throughout the plant. $^{21,22}$ Furthermore, targeted delivery to chloroplasts is known. ${ }^{23}$ Some studies report the utilization of NCs to overcome nutrient deficiencies, ${ }^{24,25}$ for growth regulation ${ }^{26}$ and for the sustained release of herbicides, ${ }^{27}$ nematocides, ${ }^{28}$ fungicides, ${ }^{29}$ and insecticides. ${ }^{30}$ Sustained and targeted delivery, efficient uptake of nutrients, and reduced environmental impacts are among the main advantages of using nano- and micro-carriers in agriculture. $^{31,32}$

Received: April 3, 2020

Revised: June 15, 2020

Published: June 16, 2020 
Scheme 1. Concept of the NC-Mediated Drug Delivery in Plants ${ }^{a}$

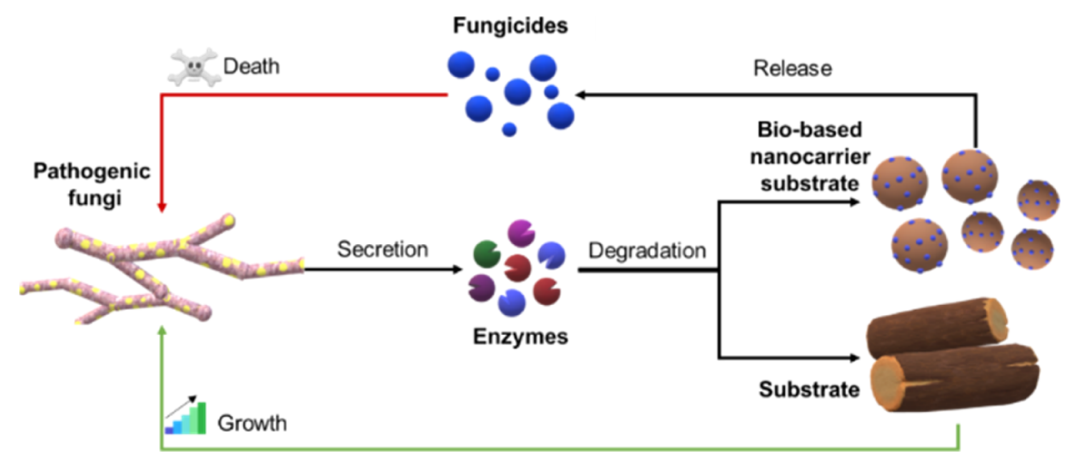

${ }^{a}$ Pathogenic fungi degrade the host plant by secreting wood-decaying enzymes, which lead to further growth of the fungal mycelium and eventually the death of the plant (green pathway). By injection of the drug-loaded NCs, a negative feedback loop is initiated as the lignin-degrading enzymes secreted by the fungi release the fungicide (red pathway).

Scheme 2. Preparation of Cross-Linked and Fungicide-Loaded Lignin NCs via the Aza-Michael Polyaddition in Miniemulsion (the Chemical Structures of the Cross-Linkers EDBEA, Spermidine, and Spermine Are Shown, Together with Their Reaction Mechanism with Methacrylated Lignin)

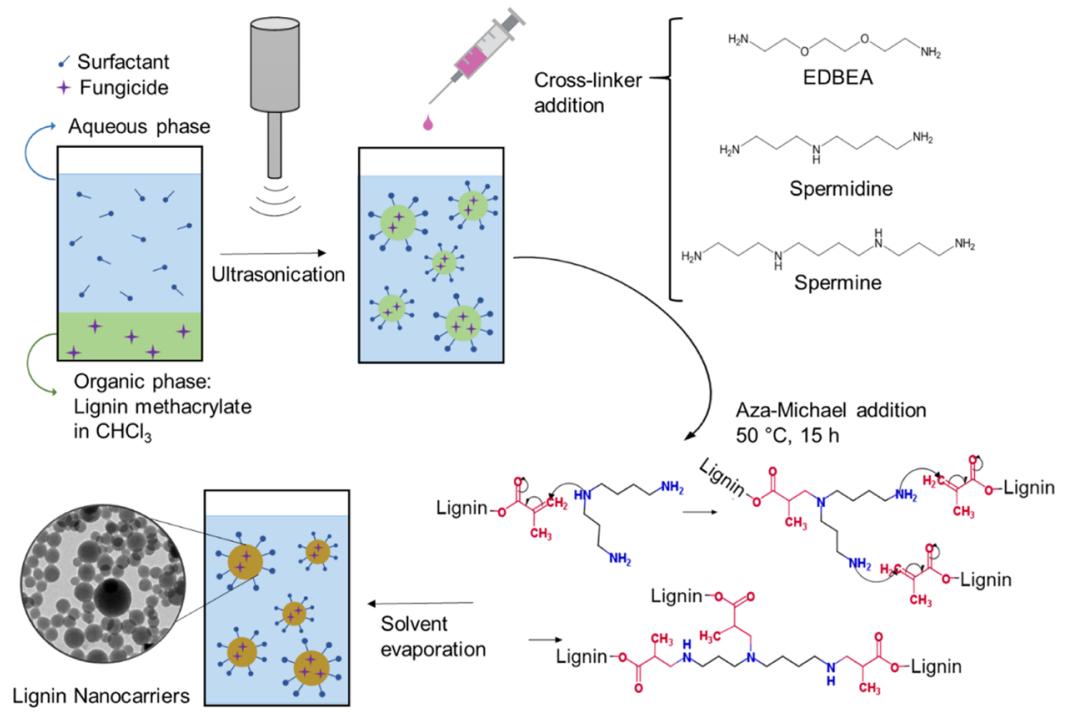

Herein, we present the preparation of lignin-derived NCs synthesized by aza-Michael addition in miniemulsion with biobased amine cross-linkers, spermidine and spermine, which were loaded efficiently with different fungicides. Such drugloaded NCs are promising for drug delivery in agriculture to reduce the amount of sprayed pesticides and to selectively release drugs by an enzymatic stimulus. ${ }^{33}$ To be applied in plant protection, the aqueous dispersion can be injected directly into the trunk of the plants to act as a negative feedback to the growing pathogen by release of the cargo upon biodegradation (Scheme 1). ${ }^{2}$ Although the utilization of NCs for delivery systems in plants is still little explored, bio-based NCs portray a promising prospect for the sustainable administration of agrochemicals. In our previous study, we prepared lignin NCs (loaded with the fungicide pyraclostrobin) and studied their effect against Esca in planta, where nanoformulations delivered a superior performance by reducing the cumulative amount of fungicide in $360 \%$ over 4 years in comparison to commercial F500 formulation. ${ }^{2}$ Esca is a worldwide occurring fungal infection that has been described as one of the most destructive grapevine trunk diseases and can induce severe decline, dieback, and ultimately death of grapevine plants, which cannot be cured to date by other techniques. $^{34,35}$

In this work, we demonstrate the versatility of the lignin platform for the encapsulation of various fungicides, which are currently applied on the multiton scale in spraying formulations. We further expand the cross-linking chemistry to fully bio-based reagents by using spermine and spermidine as bio-based and biodegradable cross-linkers with additional amine functionalities that can lead to higher encapsulation efficiency (EE) of the cargo. The versatile drug load allows the use of a mix of different fungicides during the treatment, in order to prevent resistance against a single fungicide, which is a strategy applied in conventional spraying applications and becomes now available for in planta treatment. ${ }^{36}$ With several fungicides encapsulated inside of the lignin NCs, the application of drug combinations or the use of different fungicides in different (consecutive) years becomes feasible to treat Esca but also other fungal plant diseases. Finally, we proved the efficient treatment of Vitis vinifera plants by injection of boscalid-loaded NCs and monitored their development over a period of 4 years, proving a drastic reduction in Esca leaf symptoms after a single injection in the 
first year of the study. Taken together, lignin nano- and microcarriers are a versatile platform for modern drug delivery in agriculture and might help to decrease the use of extensive spraying of fungicides.

\section{EXPERIMENTAL SECTION}

Materials. Kraft lignin (total hydroxyl group content: $6.13 \mathrm{mmol} \mathrm{g}^{-1}$ determined by ${ }^{31} \mathrm{P}$ NMR spectroscopy ${ }^{37}$ ), methacrylic anhydride, triethylamine, 2,2' (ethylenedioxy)bis(ethylamine)EDBEA, spermine, spermidine, lithium chloride, 2-chloro-4,4,5,5tetramethyl-1,3,2-dioxaphospholane, endo- $N$-hydroxy-5-norbornene2,3-dicarboximide, pyridine, deuterated chloroform, and chromium(III)acetylacetonate, tebuconazole, and boscalid were purchased from Sigma-Aldrich and used as received. Azoxystrobin and pyraclostrobin were purchased from TRC, Canada. Dimethylformamide (DMF) and isopropanol were obtained from Merck. Kraft lignin and lithium chloride were dried overnight at $70{ }^{\circ} \mathrm{C}$ in a vacuum oven before use. The anionic surfactant sodium dodecyl sulfate (SDS) was purchased from Alfa Aesar and used as received.

Lignin Methacrylate. Lignin was modified by esterification with methacrylic anhydride (Scheme S1) as described in previous work. ${ }^{2}$ Kraft lignin (2 g, number of hydroxyl groups: $12.26 \mathrm{mmol}$ ) was dissolved in $60 \mathrm{~mL}$ of $\mathrm{LiCl} / \mathrm{DMF}$ at $90{ }^{\circ} \mathrm{C}$ under argon. After complete dissolution, $1 \mathrm{~mL}$ of triethylamine $(10 \mathrm{mmol})$ was added to the lignin solution and stirred for $15 \mathrm{~min}$ at $50{ }^{\circ} \mathrm{C}$. Methacrylic anhydride $(3 \mathrm{~mL}, 20 \mathrm{mmol})$ was then slowly injected into the reaction flask. The reaction was maintained at $50{ }^{\circ} \mathrm{C}$ overnight. The reaction mixture was precipitated into isopropanol, and the solid was isolated by centrifugation at $3000 \mathrm{rpm}$. The product was repeatedly dissolved in chloroform and precipitated in isopropyl alcohol three times. The product was dried at room temperature in a vacuum oven. Yields are typical ca. 50\%.

Preparation of NCs. The preparation of lignin NCs was carried out by miniemulsion polymerization followed by solvent evaporation (Scheme 2). The typical procedure is the following: ligninmethacrylate (MA) $(50 \mathrm{mg}, 4.6 \mu \mathrm{mol})$ was dissolved in $500 \mathrm{mg}$ of chloroform. This solution was added into an aqueous solution (4.5 $\mathrm{mL}$ ) containing $0.1 \mathrm{wt} \%$ of the surfactant SDS, and the resulting mixture was pre-emulsified under vigorous stirring using an Ultra Turrax homogenizer at $20,000 \mathrm{rpm}$ for $1 \mathrm{~min}$ under ice-cooling to prevent evaporation of the organic solvent. The pre-emulsion was submitted to ultrasonication for $3 \mathrm{~min}(1 / 2 \mathrm{in}$. tip, $70 \%$ amplitude, 20 $\mathrm{s}$ pulse on followed by $10 \mathrm{~s}$ pulse off) under ice-cooling in order to prevent evaporation of the solvent and the polymerization of ligninMA due to heating. After the formation of a stable miniemulsion, an aqueous solution $(0.5 \mathrm{~mL})$ containing the cross-linker-EDBEA (25 $\mathrm{mg})$; spermine $(17 \mathrm{mg})$; or spermidine $(16 \mathrm{mg})$ - was added dropwise. Even though cross-linkers with different numbers of amine functionalities were used, the ratio $\mathrm{NH} / \mathrm{MA}$ was maintained the same and thus the difference in the mass of cross-linker added to the miniemulsion. After the addition of the cross-linker, the reaction was carried out for $15 \mathrm{~h}$ at $50{ }^{\circ} \mathrm{C}$ under mild stirring. After the completion of the polymerization process, chloroform was evaporated by stirring the open vessel overnight at room temperature, and the amount of water loss in the process was refilled to $5 \mathrm{~mL}$. The same protocol was successful by upscaling to $50 \mathrm{~mL}$ of final volume.

In order to encapsulate a set of drugs to treat fungal infections, fungicides were dissolved at different concentrations in the organic phase together with the lignin-MA and chloroform and the procedure followed as described above. Pyraclostrobin, azoxystrobin, and tebuconazole amounts were typically 10 and $15 \mathrm{mg}$, and boscalid amounts were $5 \mathrm{mg}$ because of lower solubility in $\mathrm{CHCl}_{3}$.

Dynamic Light Scattering. The hydrodynamic diameters of the particles were measured by dynamic light scattering (DLS) with a Zetasizer Nano S90 submicron particle sizer (Malvern Panalytical, $\mathrm{UK})$ at a fixed angle of $90^{\circ}$ and a laser diode running at $633 \mathrm{~nm}$; samples were diluted before measurement.

Gel Permeation Chromatography. For gel permeation chromatography measurements in DMF (containing $0.25 \mathrm{~g} \cdot \mathrm{L}-1$ of lithium bromide as an additive), an Agilent 1100 Series (Agilent Technologies 1260 Infinity) was used as an integrated instrument, including PSS GRAM columns $(1000 / 1000 / 100 \mathrm{~g})$, a UV detector $(270 \mathrm{~nm})$, and an RI detector at a flow rate of $1 \mathrm{~mL} / \mathrm{min}$ at $60{ }^{\circ} \mathrm{C}$. Calibration was carried out using PS standards provided by Polymer Standards Service.

Electron Microscopy. For NC detection, a JEOL 1400 transmission electron microscope (TEM) with a $\mathrm{LaB}_{6}$ cathode (JEOL GmbH, Eching, Germany) was used. The copper grid had been modified with a carbon film (200 mesh, Science Services, Munich, Germany) before the TEM specimen was prepared. Therefore, the dispersion was drop-cast on a copper grid. After drying of the TEM grid at room temperature, it was inserted into a sample holder and transferred into the TEM. The TEM was operated at an acceleration voltage of $120 \mathrm{kV}$.

NMR Spectroscopy. ${ }^{1} \mathrm{H}$ and ${ }^{31} \mathrm{P}$ nuclear magnetic resonance (NMR) spectroscopy was performed at a Bruker AVANCE (USA) system at $300 \mathrm{MHz}$. For ${ }^{1} \mathrm{H}$ NMR spectroscopy, $5 \mathrm{mg}$ of the sample was dissolved in $600 \mu \mathrm{L}$ of DMSO- $d_{6}$. To determine the number of $\mathrm{OH}$ groups in the sample, ${ }^{31} \mathrm{P}$ NMR spectroscopy was used after derivatization according to the literature: ${ }^{37} 20 \mathrm{mg}$ of dried sample was dissolved in $550 \mu \mathrm{L}$ of a $\mathrm{CDCl}_{3}$-pyridine mixture (4/6 v/v ratio) in the presence of the internal standard endo- $N$-hydroxy-5-norbornene2,3-dicarboximide and the relaxation agent $\mathrm{Cr}$ (III)acetylacetonate in an NMR tube. 2-chloro-4,4,5,5-tetramethyl-1,3,2-dioxaphospholane $(100 \mu \mathrm{L})$ was added, and ${ }^{31} \mathrm{P}$ NMR spectra were recorded.

IR Spectroscopy. To investigate the chemical structures of lignin, lignin-MA, and cross-linked NCs, their Fourier transform infrared (FTIR) spectra were recorded by Nicolet iS10 with a vertical ATR accessory. Lignin and lignin-MA samples were dried at room temperature in the vacuum oven, while the $\mathrm{NCs}$ were extracted from the latex by centrifugation, at $10,000 \mathrm{rpm}$ for $60 \mathrm{~min}$, washed twice with water to remove any water-soluble impurities, and dried in a vacuum oven at $50{ }^{\circ} \mathrm{C}$ overnight. Spectra were recorded between 600 and $4000 \mathrm{~cm}^{-1}$ at a resolution of $4 \mathrm{~cm}^{-1}$ and coding 32 scans.

Encapsulation Efficiency. In order to determine the EE, the amount of fungicide loaded in the NCs, high-performance liquid chromatography (HPLC) was used to quantify the amount of nonencapsulated fungicides from the latex after centrifugation $(10,000$ $\mathrm{rpm}$ for $60 \mathrm{~min}$ ) and thereof estimate the EE according. The HPLC measurements were performed on an Agilent Technologies Series 1200 setup equipped with a UV detector (pyraclostrobin at $280 \mathrm{~nm}$, azoxystrobin at $260 \mathrm{~nm}$, tebuconazole at $220 \mathrm{~nm}$, and boscalid at 280 $\mathrm{nm}$ ) and an ELSD detector 385-LC (both Agilent Technologies, USA). The analyses of tebuconazole, pyraclostrobin, and boscalid were done using an Agilent Eclipse Plus C18 and an eluent gradient from $\mathrm{THF} /$ water $+0.1 \%$ FA $20 / 80$ to $100 / 0$. In addition, the analyses of azoxystrobin were conducted using a Nucleodur Gavity SB C18 and the same eluent gradient. The EE and the weight percentage of pyraclostrobin $n$ NCs were determined according to the following eq

$$
\mathrm{EE}(\%)=\frac{m(\text { fungicide initial })-m(\text { fungicide supernatant })}{m(\text { fungicide initial })} \times 100 \%
$$

Antifungal Activity. Phaeomoniella chlamydospora and Phaeoacremonium minimum were used for the spore germination test as previously published. ${ }^{2}$ The tested compounds and nanoparticles were prepared in aliquots of $1,5,10,25$, and $50 \mu \mathrm{g} \cdot \mathrm{mL}^{-1}$ (active ingredient) per 96-well dissolved in $\mathrm{H}_{2} \mathrm{O}$. The spores were diluted in yeast glucose mineral medium to a final concentration of 2000 spores $/ \mathrm{mL}$. The spore suspension $(200 \mu \mathrm{L})$ was added to the nanoparticles. The plates were incubated at $27^{\circ} \mathrm{C}$ and $120 \mathrm{rpm}$ on an incubator, and the optical density (OD) was measured every $24 \mathrm{~h}$ at a wavelength of $600 \mathrm{~nm}$. The antifungal activity was obtained by comparing the OD after the incubation of samples treated with the positive control (just water is added), negative control (plain fungicide is added), and NC dispersion. The OD relative to the positive control was considered $100 \%$ of fungi growth. 
Trunk Injections (According to a Previously Established Protocol ${ }^{2}$ ). The site of the trunk that was selected for the injection, and a ca. $8 \mathrm{~mm}$ deep and $6 \mathrm{~mm}$ wide hole was drilled into the grapevine trunk. The chosen plants had trunk diameters of at least 20 $\mathrm{mm}$. The drill head was sterilized in $70 \%$ ethanol for $1 \mathrm{~min}$ before the next plant was treated. After the drilling, the injection system [supplied from Tree Tech Microinjection Systems (FL, US)] was filled with $5 \mathrm{~mL}$ of the lignin NC dispersion (empty NCs or boscalidloaded NCs) and inserted to the grapevine trunk hole. The scroll spring pressure system was pushed after the injector was placed safely in the trunk, and the dispersion was allowed to flow into the plant. After $24 \mathrm{~h}$, the injection system was removed, and the wound was sealed with grafting wax. The plants were monitored for increasing or decreasing foliar Esca symptoms in comparison to their appearance to the control plants. The discolorations were documented using a digital camera. The plants were monitored in the following years for Esca leaf symptoms.

\section{RESULTS AND DISCUSSION}

Lignin-MA Synthesis. Kraft lignin was modified into lignin-MA by esterification of the phenolic and aliphatic hydroxyls with methacrylic anhydride (Scheme S1). A degree of functionalization of ca. $90 \%$ was calculated by a derivatization method with 2-chloro-4,4,5,5-tetramethyl-1,3,2dioxaphospholane via ${ }^{31} \mathrm{P}$ NMR spectroscopy as described previously (eq S1). ${ }^{37}$ The ${ }^{31} \mathrm{P}$ NMR spectra (Figure 1a) proved a high amount of phenolic and aliphatic OH-groups in the neat Kraft lignin, which were drastically reduced after methacrylation. The ${ }^{1} \mathrm{H}$ NMR spectrum of the methacrylated lignin (Figure S1) showed resonances at 5.75 and $6.25 \mathrm{ppm}$ for the hydrogens of the double bonds in the MA groups. The successful modification was also confirmed in the IR spectra
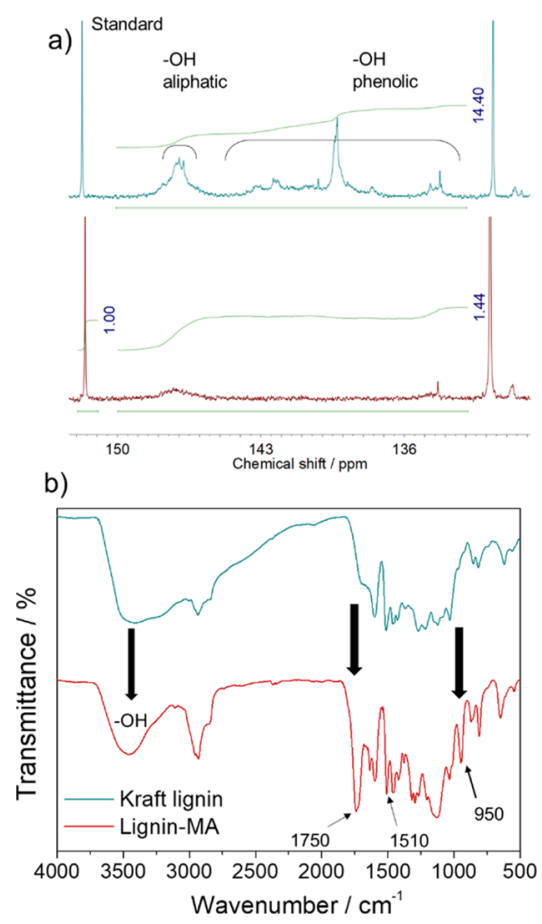

Figure 1. (a) Determination of the degree of functionalization of lignin by a ${ }^{31} \mathrm{P}$ NMR derivatization method. ${ }^{37}$ Top: Kraft lignin and bottom: lignin-MA after derivatization; the phenolic and aliphatic hydroxyls are indicated in the figure compared to the standard. The values of the integrals are indicated in each spectrum. Methacrylation degree ca. 93\%. (b) FT-IR spectra of Kraft lignin and lignin-MA normalized in relation to the benzene ring stretch at $1510 \mathrm{~cm}^{-1}$.
(Figure $1 \mathrm{~b}$ ). The spectra were normalized in relation to the benzene ring stretch band of native lignin at $1510 \mathrm{~cm}^{-1}$ : the decrease of the $\mathrm{OH}$ band at ca. $3500 \mathrm{~cm}^{-1}$ and the appearance of ester $\mathrm{C}=\mathrm{O}$ and vinyl $\mathrm{C}=\mathrm{C}$ signals at 1750 and $950 \mathrm{~cm}^{-1}$ after the reaction further underlines the successful attachment of MAs.

Lignin was analyzed by SEC both prior to and after chemical modification-molecular weight distribution curves can be found in the Supporting Information and data summed up in Table S1. Apparent $M_{\mathrm{w}}$ values calculated based on retention time compared to a PS standard in $\mathrm{DMF} / \mathrm{LiBr}$ increased from 3.1 to $25.4 \mathrm{kDa}$ after modification.

NC Preparation. Lignin-based NCs were prepared by cross-linking the methacrylic double bonds of lignin-MA with amine cross-linkers by aza-Michael addition in miniemulsion. Aza-Michael addition is a nucleophilic conjugate addition reaction between an amine and a $\pi$-activated compound and may be considered a click reaction. ${ }^{38}$ Because of the absence of additional catalysts, tolerance to water, and typically very high yields, it is ideal for preparing cross-linked lignin NC. The biobased spermine and spermidine makes it additionally environmentally friendly. ${ }^{39}$ NCs were prepared using the miniemulsion technique, in which nanodroplets of a (macro)monomeric phase are dispersed in a continuous aqueous phase. ${ }^{40}$ The miniemulsion was prepared prior to polymerization by high shear forces with an ultrasonic tip, which breaks down the disperse phase into nanodroplets, typically droplet size range from 50 to $500 \mathrm{~nm}$, depending on the conditions applied during the preparation. ${ }^{40,41}$ In our case, Scheme 2 summarizes the protocol that was used to prepare the aqueous lignin NC dispersions, loaded with fungicides as well as a scheme of the mechanism of the aza-Michael addition reaction and the chemical structures of the synthetic cross-linker (EDBEA) and the two bio-based cross-linkers (spermidine and spermine).

After the polymerization, the lignin NC dispersions were characterized by DLS and TEM with respect to diameters and polydispersities (Table 1 and Figure 2). Under these

\section{Table 1. Data for Lignin-Based NCs ${ }^{a}$}

$\begin{array}{clccc}\text { entry } & \text { cross-linker } & D_{\mathrm{i}} / \mathrm{nm} & \text { PDI } & \zeta \text {-potential } / \mathrm{mV} \\ \text { LNP01 } & \text { EDBEA } & 170 & 0.12 \pm 0.04 & -20 \\ \text { LNP02 } & \text { spermidine } & 230 & 0.11 \pm 0.03 & -12 \\ \text { LNP03 } & \text { spermine } & 220 & 0.14 \pm 0.04 & -10\end{array}$

${ }^{a}$ Diameters and PDI obtained by DLS and $\zeta$-potential. Errors are the standard deviation of triplicates.

conditions, the average particle diameter was found to be ca. $200 \mathrm{~nm}$ with a polydispersity index (PDI) of ca. 0.1 for all cross-linkers used. Some coagulation was observed after some hours of reaction, when spermine was used as cross-linker (LNP03); however, the coagulum was removed by filtration through a Kimberly-Cark precision wipe after the reaction. Apart from that, no precipitation, coagulation, or flocculation of the dispersions was observed, which typically had solid contents of $1.2-1.5 \%$. The dispersions were stabilized with the anionic surfactant sodium dodecyl sulfate (SDS) as the surfactant and exhibited negative $\zeta$-potentials (at $\mathrm{pH} 8$ ) between -10 and $-20 \mathrm{mV}$ (Table 1). The different $\zeta$ potentials could be rationalized by the presence of additional amine groups from spermine, which might counterbalance some of the negatively charged SDS. 

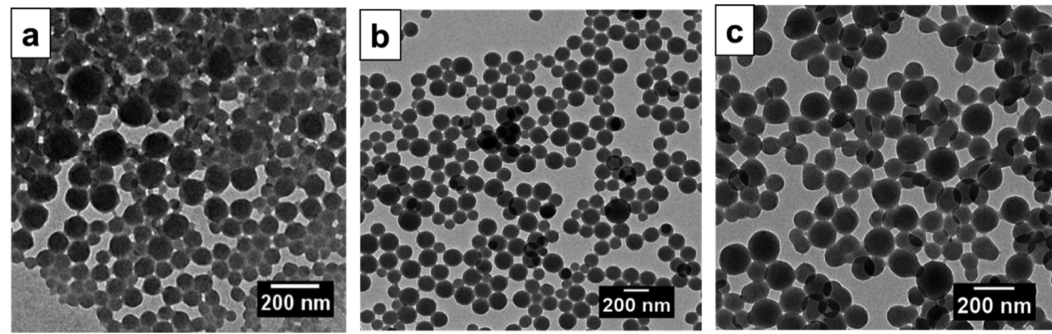

Figure 2. TEM images of the cross-linked lignin NCs according to the cross-linkers used (a) EDBA, (b) spermidine, and (c) spermine.

Particle size and morphology of the lignin NCs after crosslinking polymerization were further investigated by TEM (Figure 2). NCs with a solid spherical morphology were obtained; the diameters from TEM were smaller compared to DLS values, which indicates a certain swelling in dispersion. The chemical functionalities of the lignin NCs and the doublebond conversion were analyzed by IR spectroscopy. The spectra (Figure 3) were normalized in relation to the benzene

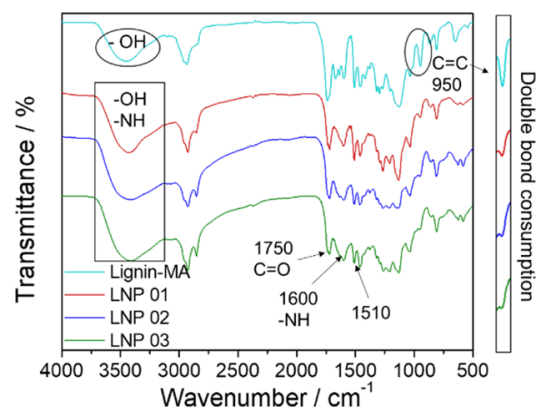

Figure 3. Overlay of the FTIR spectra of lignin-MA (cyan, top) and lignin NCs after aza-Michael addition in miniemulsion with different cross-linkers [LNP 01-EDBEA (red), LNP 02-spermidine (blue), and LNP 03-spermine (green)].

ring stretch band of native lignin at $1510 \mathrm{~cm}^{-1}$. The decrease of $\mathrm{C}=\mathrm{C}$ bands of the vinyl groups at $950 \mathrm{~cm}^{-1}$ due to their consumption during the aza-Michael cross-linking polymerization and the appearance of $-\mathrm{NH}$ bands at ca. $1500 \mathrm{~cm}^{-1}$ in the cross-linked lignin NCs at ca. $3500 \mathrm{~cm}^{-1}$ can be used to monitor the reaction progress.

Drug Loading. This synthetic protocol was used to prepare a library of fungicide-loaded lignin-based NCs. Different amounts of the fungicides (up to $30 \mathrm{wt} \%$ of the final NC) were dissolved in the dispersed phase together with the methacrylated lignin and encapsulated in situ via aza-Michael polyaddition. We investigated pyraclostrobin, boscalid, azoxystrobin, and tebuconazole as a set of fungicides because of their high solubility in organic solvents and low solubility in water. These fungicides are currently used in spraying applications in agriculture against different fungal diseases either alone or in mixed formulations due to their different modes of action (Figure S3 shows their chemical structures). ${ }^{24,25}$ Herein, we studied the in vitro activity against fungi, related to the grapevine trunk disease Esca. ${ }^{34,35}$

The concentrations of the drugs were varied from 10 to 30 wt \% (Tables 2 and S2, also sample nomenclature is explained in the Table's caption), which is related to $1-3 \mathrm{mg}$ of drug per $\mathrm{mL}$ of the dispersion. Boscalid, however, had a lower solubility under these conditions compared to the other drugs; therefore, the maximum concentration of boscalid was set to $1 \mathrm{mg} \cdot \mathrm{mL}^{-1}$. The particle diameters determined by DLS ranged typically between 200 and $300 \mathrm{~nm}$ with moderate PDI (Table 2); samples AS01 and PS01 exhibited higher diameters with increased PDI, probably because of the presence of some aggregates, which could not be separated by filtration or centrifugation [DLS measurements can be found in the Supporting Information (Figures S12 and S13)]. TEM images showed particle diameters in the range of 100-300 nm (Figures 4 and S9), indicating some aggregation, which do not pose an impediment to the application of these NCs inside of plants, as the channels of xylem/phloem have diameters of several micrometers. ${ }^{26}$

All drugs proved encapsulation efficiencies of at least $70 \%$ to almost quantitative (Figure 5 and Table 2). Interestingly, for all samples using spermidine as the cross-linker, a higher EE compared to the previously reported EDBEA was found, indicating a higher degree of cross-linking. In addition, we proved that no leaking from the particles was observed after centrifugation and redispersion in deionized water. The dispersions were stirred for $48 \mathrm{~h}$ and then centrifuged again; the supernatant was analyzed by HPLC proving almost no

Table 2. Drug-Loaded Lignin NCs According to Cross-Linkers Utilized ${ }^{a}$

\begin{tabular}{|c|c|c|c|c|c|c|}
\hline entry & drug & $\operatorname{drug} / \mathrm{mg} \cdot \mathrm{mL}^{-1}$ & cross-linker & $D_{\mathrm{p}} / \mathrm{nm}$ & PDI & $\mathrm{EE} / \%$ \\
\hline AE01 & azoxystrobin & 2 & EDBEA & 190 & $0.17 \pm 0.06$ & 88 \\
\hline AS01 & & 2 & spermidine & $(470)$ & $(0.33 \pm 0.09)$ & 99 \\
\hline BE01 & boscalid & 1 & EDBEA & 210 & $0.12 \pm 0.03$ & 77 \\
\hline BS01 & & 1 & spermidine & 225 & $0.14 \pm 0.03$ & 98 \\
\hline PE01 & pyraclostrobin & 2 & EDBEA & 206 & $0.3 \pm 0.1$ & 93 \\
\hline PS01 & & 2 & spermidine & $(535 \pm 155)$ & $(0.4 \pm 0.1)$ & $>99$ \\
\hline TE01 & tebuconazole & 2 & EDBEA & 165 & $0.19 \pm 0.04$ & 73 \\
\hline TS01 & & 2 & spermidine & 300 & $0.2 \pm 0.1$ & 97 \\
\hline
\end{tabular}

${ }^{a}$ Average particle size $\left(D_{\mathrm{p}}\right)$ and PDI measured by DLS (measurements presented as mean \pm SD of triplicates; values in brackets exhibited some aggregation) and EE measured by HPLC. Sample nomenclature: first letter denotes the drug $(\mathrm{A}=$ azoxystrobin, $\mathrm{B}=\mathrm{boscalid}$, $\mathrm{P}=$ pyraclostrobin, $\mathrm{T}$ = tebuconalzole; second letter denotes the cross-linker: $\mathrm{E}=\mathrm{EDBEA}, \mathrm{S}=$ spermidine, number $=$ number of sample). 

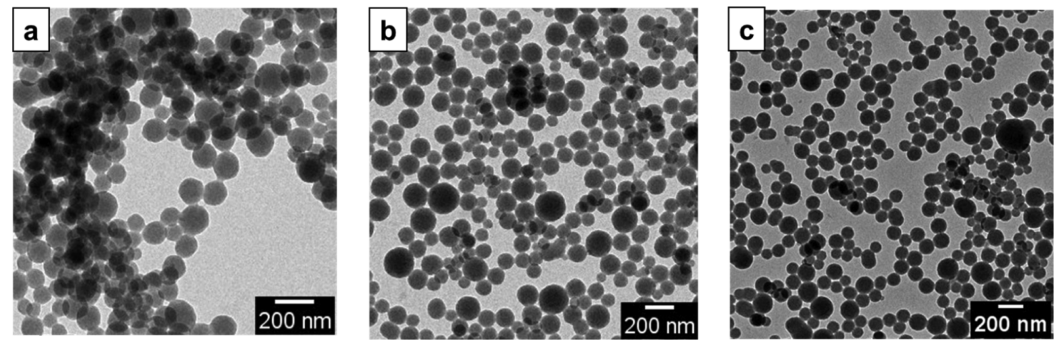

Figure 4. Drug-loaded lignin NCs cross-linked with spermidine in miniemulsion: (a) azoxystrobin (AS01), (b) pyraclostrobin (PS01), and (c) tebuconazole (TS01).

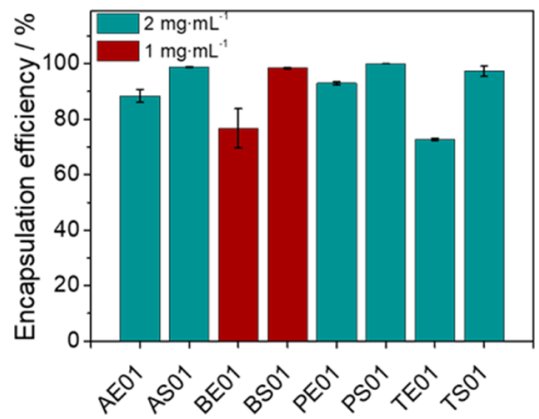

Figure 5. Detected encapsulation efficiencies (by HPLC) of fungicide-loaded lignin NCs after one washing step [sample description: NCs containing azoxystrobin (AE01 and AS01), boscalid (BE01 and BS01), pyraclostrobin (PE01 and PS01), and tebuconazole (TE01 and TS01)]. Samples in red contained $1 \mathrm{mg}$ of fungicide per $\mathrm{mL}$ of dispersion, whereas samples in blue contained 2 $\mathrm{mg} \cdot \mathrm{mL}^{-1}$. Error bars are the standard deviation of triplicates.

release of the drugs from the lignin carriers $(<5 \%$ for samples: AE02, AS02, BS01, PE02, PS02, and TS02), regardless which cross-linker was used during the preparation (Figure S10).

Antifungal Activity. Dose-dependent antifungal activities of NC formulations were compared to those of free fungicides by means of minimum inhibitory concentration (MIC) for a series of test microorganisms, all producing lignases: $P$. chlamydospore, Neonectria ditissima, Phytophtora infestans, Magnoporthe oryzae, Botrytis cinerea, Neofusicoccum parvum (Table 3). We studied both the pure fungicides (azoxystrobin, boscalid, pyraclostrobin, and tebuconazole) and the encapsulated fungicides (samples: AE02, BE01, PE02, TE02, and the mixture PE02/BE01). For the MIC test, the fungicide concentrations were $5,10,25$, and $50 \mu \mathrm{g} / \mathrm{mL}$ for bulk and nanoformulations. Most of the encapsulated fungicides in lignin NCs presented lower MICs compared to their bulk counterparts (Table 3). This might be explained by the higher availability of the encapsulated drugs in the lignin carrier dispersions, which increased the antifungal activity. Further, the NCs were dispersed in water, whereas the low solubility of the pure fungicides in water might decrease the antifungal activity of bulk formulations in aqueous media, while the lignin NCs can be degraded by the organisms, followed by the release of the fungicides. ${ }^{2}$

Spore germination inhibition and fungal growth inhibition in vitro were further tested against Esca-associated fungi, $P$. chlamydospora, and P. minimum. The NC formulations were incubated at a final concentration of $25 \mu \mathrm{g} / \mathrm{mL}$ in relation to the active compound (fungicide), together with a mixed culture of $P$. chlamydospora and P. minimum spores; after $96 \mathrm{~h}$, the growth inhibition activity was determined by the OD of the media at $600 \mathrm{~nm}$.

The growth was normalized in relation to the value relative to the positive control, which was set to $100 \%$ growth. The results underline the antifungal activity of the drug-loaded lignin NCs that inhibited the growth of the fungal mycelium similar to the free drugs under these conditions (Figure 6a). Increasing the drug concentration in the dispersions to $3 \mathrm{mg}$. $\mathrm{mL}^{-1}$ did not further decrease the fungal growth (Figure $6 \mathrm{~b}$ ), which proved results similar to those with $2 \mathrm{mg} \cdot \mathrm{mL}^{-1}$. Furthermore, drug mixtures of azoxystrobin and boscalid, azoxystrobin and tebuconazole, and tebuconazole and boscalid efficiently inhibited fungi growth in more than $60 \%$ even though the concentration of each drug in the mixture was reduced compared to the dispersions containing each drug separately. Drug mixtures might be beneficial for the use in the fields to prevent resistances of plant diseases against single fungicide formulation, which are currently applied in spraying applications. $^{25}$ Boscalid has not shown activity, in either bulk or nanoencapsulated, in the germination assay against $P$. chlamydospora and P. minimum spores. However, boscalid nanoformulations were able to inhibit fungal growth in MIC tests (Table 3 ), probably because MIC assays are performed with 18-day-old fungi while germination and mycelial growth

Table 3. Dose-Dependent Antifungal Activities of NC Formulations and Bulk Fungicide Formulations ${ }^{a}$

\begin{tabular}{|c|c|c|c|c|c|c|c|c|c|}
\hline \multirow[b]{2}{*}{ test organism } & \multicolumn{9}{|c|}{$\mathrm{MIC} / \mu \mathrm{g} / \mathrm{mL}$} \\
\hline & AE02 & BE01 & PE02 & TE02 & PE02/BE01 & Azox. & Bos. & Pyr. & Teb. \\
\hline P. chlamydospora & 5 & 5 & 5 & 5 & 5 & 10 & 10 & 10 & 10 \\
\hline N. ditissima & 25 & $>50$ & 5 & 5 & 10 & 50 & $>50$ & 50 & $>50$ \\
\hline P. infestans & 50 & 50 & 5 & 5 & 25 & $>50$ & $>50$ & 50 & 10 \\
\hline M. oryzae & 25 & $>50$ & 5 & 25 & 5 & 50 & 50 & 10 & 50 \\
\hline B. cinerea & 10 & 10 & 5 & 50 & 5 & 10 & 10 & 10 & 50 \\
\hline N. parvum & 5 & $>50$ & 5 & $>50$ & 5 & 5 & $>50$ & 5 & $>50$ \\
\hline
\end{tabular}

${ }^{a}$ MIC toward P. chlamydospora, N. ditissima, P. infestans, M. oryzae, B. cinerea, and N. parvum. MIC (active ingredient, $\mu \mathrm{g} / \mathrm{mL}$ ) represents the concentration at which inhibition of fungal growth was first observed. 

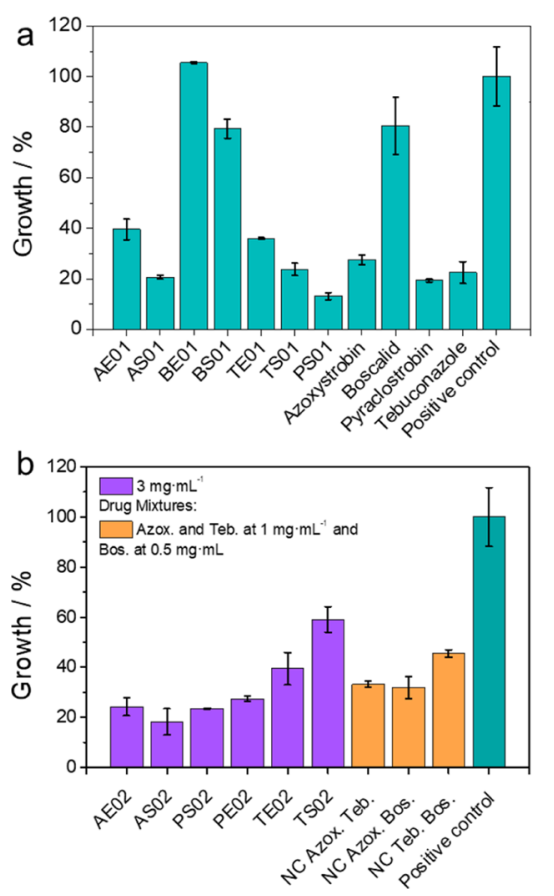

Figure 6. Spore germination and mycelial growth of $P$. chlamydospora and P. minimum. The NC dispersions were incubated with the spores for $96 \mathrm{~h}$. Positive control consists of a spore sample without NCs. Free drugs were tested for comparison. Error bars are the standard deviation from triplicates. (a) NCs containing azoxystrobin (AE01 and AS01), boscalid (BE01 and BS01), pyraclostrobin (PE01 and PS01), and tebuconazole (TE01 and TS01). Boscalid samples contain $1 \mathrm{mg}$ of fungicide per $\mathrm{mL}$ of dispersion, while the remaining samples contain $2 \mathrm{mg} \cdot \mathrm{mL}^{-1}$. (b) NCs containing 30 wt \% of azoxystrobin (AE02 and AS02), pyraclostrobin (PE02 and PS02), and tebuconazole (TE02 and TS02) and drug mixtures of azoxystrobin (10 wt \%), tebuconazole (10 wt \%), and boscalid (5 wt \%). The crosslinker used, EDBEA or spermidine, is denoted in the sample denomination by the letter $\mathrm{E}$ or $\mathrm{S}$, respectively.

assays are performed with conidia (mitospores). Furthermore, additional in planta studies using Boscalid-loaded NCs proved their effectiveness over a period of at least 4 years (cf. Figure 7). In vitro tests were conducted to assure the efficiency of the NCs to inhibit the growth of fungal mycelium; because of the direct application of NCs to the culture, a quick release of the cargo upon $\mathrm{NC}$ degradation was expected. In contrast, in vivo studies were performed to test the long-term antifungal effect over several years, which might be attributed to a slower degradation in planta or a possible depot effect that lasts for several years. Each of four V. vinifera L. cv. "Portugieser" plants was treated with $5 \mathrm{~mL}$ of a $1 \mathrm{wt} \%$ dispersion of lignin NCs using a trunk injector provided by Tree Tech; both empty and boscalid-loaded NCs were monitored over a period of 4 years after a single injection in 2015 until summer 2019, according to scoring procedures as published recently. ${ }^{2}$ Once introduced by trunk injection, we expect that the NCs can be transported within the plant, via xylem (upward movement) and phloem (downward movement), ${ }^{21,42}$ from the injection site to reach the infected tissues. Although the Esca symptoms on leaves and grapes of the plants treated with the empty control increased during 4 years of monitoring, almost no signs of Esca were detected in the boscalid-NC treated plants. In summary, these data prove the versatility of the lignin encapsulation of various fungicides against Esca associated fungi.

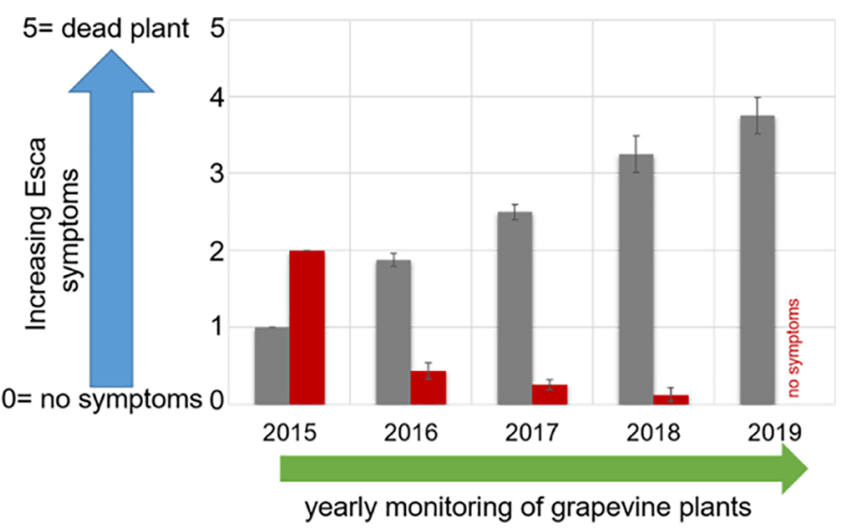

Figure 7. In planta application of lignin NCs. Plot showing the severity of Esca leaf symptoms on four Portugieser grapevine plants monitored yearly from 2015 until 2019: comparison between treatments with empty lignin NCs (i.e., without loaded fungicide, gray bars) and lignin NCs (loaded with boscalid, red bars). (Note: $y$ axis shows the severity of Esca leaf symptoms $0=$ healthy plant, no symptoms; 5 = dead plant; more details in ref 2).

\section{SUMMARY}

Lignin-based NCs with versatile drug-load were synthesized by aza-Michael cross-linking polymerization in miniemulsion of methacrylated lignin followed by solvent evaporation. Kraft lignin was submitted to chemical modification by esterification with methacrylic anhydride to produce lignin-MA with $>90 \%$ of methacrylation estimated by ${ }^{31} \mathrm{P}$ NMR. Lignin-MA underwent aza-Michael cross-linking polymerization with bio-based cross-linkers in miniemulsion to yield NCs with average particle sizes of ca. $200 \mathrm{~nm}$. The double bond conversion due to the cross-linking reaction was evaluated by FTIR. In addition, this method has proven versatility when it comes to drug loading because a set of hydrophobic fungicides were encapsulated with high efficiency $>75 \%$ estimated by HPLC. A versatile drug load is important to overcome fungicide resistance that might be developed after excessive use of agrochemicals. After NC syntheses and characterization, they were submitted to biological testing against Esca-associated fungi $P$. chlamydospora and $P$. minimum and showed promising results in fungi growth inhibition after $96 \mathrm{~h}$ in vitro and boscalid-loaded lignin NCs have shown efficient and long-term activity in in planta studies. Thus, the NCs are of high interest as biodegradable delivery vehicles to be applied by trunk injection for a broad range of agricultural applications, but especially against Esca, which is a devastating trunk disease in grapevines.

\section{ASSOCIATED CONTENT}

\section{sI Supporting Information}

The Supporting Information is available free of charge at https://pubs.acs.org/doi/10.1021/acs.biomac.0c00487.

Composition of NCs, average particle size (DLS), and $\mathrm{EE}$; chemical structures of compounds: fungicides and lignin-MA; NMR spectra; SEM and TEM images; and SEC elugrams, HPLC calibration curves, and raw data for in planta studies (PDF) 


\section{AUTHOR INFORMATION}

\section{Corresponding Authors}

Katharina Landfester - Max-Planck-Institut für Polymerforschung, 55128 Mainz, Germany; @orcid.org/ 0000-0001-9591-4638; Phone: 00496131 379581; Email: landfester@mpip-mainz.mpg.de; Fax: 00496131370 330

Frederik R. Wurm - Max-Planck-Institut für Polymerforschung, 55128 Mainz, Germany; @ orcid.org/0000-0002-69558489; Email: wurm@mpip-mainz.mpg.de

\section{Authors}

Thiago O. Machado - Max-Planck-Institut fur Polymerforschung, 55128 Mainz, Germany; Department of Chemical Engineering and Food Engineering, Federal University of Santa Catarina, Florianópolis 88040-900, Santa Catarina, Brazil

Sebastian J. Beckers - Max-Planck-Institut für Polymerforschung, 55128 Mainz, Germany

Jochen Fischer - Institute for Biotechnology and Drug Research, 67663 Kaiserslautern, Germany

Beate Müller - Max-Planck-Institut für Polymerforschung, 55128 Mainz, Germany

Claudia Sayer - Department of Chemical Engineering and Food Engineering, Federal University of Santa Catarina, Florianópolis 88040-900, Santa Catarina, Brazil; ๑ orcid.org/0000-00031044-2905

Pedro H. H. de Araújo - Department of Chemical Engineering and Food Engineering, Federal University of Santa Catarina, Florianópolis 88040-900, Santa Catarina, Brazil; ๑ orcid.org/0000-0001-5905-0158

Complete contact information is available at:

https://pubs.acs.org/10.1021/acs.biomac.0c00487

\section{Notes}

The authors declare no competing financial interest.

\section{ACKNOWLEDGMENTS}

Parts of this research were supported by the Bio-Based Industries Joint Undertaking under the European Union's Horizon 2020 research and innovation program "BioRescue" (grant agreement no. 720708). The authors thank the VW Foundation "Experiment" for funding the project "Nanoprotect". Christoph Sieber (MPI-P) is acknowledged for the TEM images. The authors are also grateful to the Coordenação de Aperfeiçoamento de Pessoal de Nivel Superior, CAPESPRINT Program, project number 88887.310560/2018-00, and to the Conselho Nacional de Desenvolvimento Cientifico e Tecnológico, CNPq.

\section{REFERENCES}

(1) Yiamsawas, D.; Beckers, S. J.; Lu, H.; Landfester, K.; Wurm, F. R. Morphology-Controlled Synthesis of Lignin Nanocarriers for Drug Delivery and Carbon Materials. ACS Biomater. Sci. Eng. 2017, 3, 2375-2383.

(2) Fischer, J.; Beckers, S. J.; Yiamsawas, D.; Thines, E.; Landfester, K.; Wurm, F. R. Targeted Drug Delivery in Plants: EnzymeResponsive Lignin Nanocarriers for the Curative Treatment of the Worldwide Grapevine Trunk Disease Esca. Adv. Sci. 2019, 6, 1802315.

(3) Yiamsawas, D.; Baier, G.; Thines, E.; Landfester, K.; Wurm, F. R. Biodegradable Lignin Nanocontainers. RSC Adv. 2014, 4, 1166111663.
(4) Lignin and Lignans as Renewable Raw Materials: Chemistry, Technology and Applications; Calvo-Flores, F. G., Dobado, J. A., IsacGarcía, J., Martín-Martínez, F. J., Eds.; John Wiley \& Sons, Ltd Registered: Chichester, U.K., 2015.

(5) Fang, Z.; Smith, R. L. In Production of Biofuels and Chemicals from Lignin; Fang, Z., Smith, R. L., Eds.; Biofuels and Biorefineries; Springer Singapore: Singapore, 2016

(6) Laurichesse, S.; Avérous, L. Chemical Modification of Lignins: Towards Biobased Polymers. Prog. Polym. Sci. 2014, 39, 1266-1290.

(7) Chen, J.; Eraghi Kazzaz, A.; AlipoorMazandarani, N.; Hosseinpour Feizi, Z.; Fatehi, P. Production of Flocculants, Adsorbents, and Dispersants from Lignin. Molecules 2018, 23, 868.

(8) Liu, D.; Sun, J.; Simmons, B. A.; Singh, S. N-Heterocyclic Carbene Promoted Decarboxylation of Lignin-Derived Aromatic Acids. ACS Sustainable Chem. Eng. 2018, 6, 7232-7238.

(9) Yan, L.; Cui, Y.; Gou, G.; Wang, Q.; Jiang, M.; Zhang, S.; Hui, D.; Gou, J.; Zhou, Z. Liquefaction of Lignin in Hot-Compressed Water to Phenolic Feedstock for the Synthesis of Phenol-Formaldehyde Resins. Composites, Part B 2017, 112, 8-14.

(10) Wurm, F. R.; Weiss, C. K. Nanoparticles from Renewable Polymers. Front. Chem. 2014, 2, 49.

(11) Dai, L.; Liu, R.; Hu, L.-Q.; Zou, Z.-F.; Si, C.-L. Lignin Nanoparticle as a Novel Green Carrier for the Efficient Delivery of Resveratrol. ACS Sustainable Chem. Eng. 2017, 5, 8241-8249.

(12) Lee, J. G.; Larive, L. L.; Valsaraj, K. T.; Bharti, B. Binding of Lignin Nanoparticles at Oil-Water Interfaces: An Ecofriendly Alternative to Oil Spill Recovery. ACS Appl. Mater. Interfaces 2018, 10, 43282-43289.

(13) Capecchi, E.; Piccinino, D.; Delfino, I.; Bollella, P.; Antiochia, R.; Saladino, R. Functionalized Tyrosinase-Lignin Nanoparticles as Sustainable Catalysts for the Oxidation of Phenols. Nanomaterials 2018, 8, 438.

(14) Beckers, S. J.; Dallo, I. A.; del Campo, I.; Rosenauer, C.; Klein, K.; Wurm, F. R. From Compost to Colloids - Valorization of Spent Mushroom Substrate. ACS Sustainable Chem. Eng. 2019, 7, 6991.

(15) Raliya, R.; Saharan, V.; Dimkpa, C.; Biswas, P. Nanofertilizer for Precision and Sustainable Agriculture: Current State and Future Perspectives. J. Agric. Food Chem. 2018, 66, 6487-6503.

(16) Kumar, S.; Nehra, M.; Dilbaghi, N.; Marrazza, G.; Hassan, A. A.; Kim, K.-H. Nano-Based Smart Pesticide Formulations: Emerging Opportunities for Agriculture. J. Controlled Release 2019, 294, 131153.

(17) Cunningham, F. J.; Goh, N. S.; Demirer, G. S.; Matos, J. L.; Landry, M. P. Nanoparticle-Mediated Delivery towards Advancing Plant Genetic Engineering. Trends Biotechnol. 2018, 36, 882-897.

(18) Husen, A.; Iqbal, M. Nanomaterials and Plant Potential: An Overview; Springer, 2019.

(19) Kumari, S.; Kumaraswamy, R. V.; Choudhary, R. C.; Sharma, S. S.; Pal, A.; Raliya, R.; Biswas, P.; Saharan, V. Thymol Nanoemulsion Exhibits Potential Antibacterial Activity against Bacterial Pustule Disease and Growth Promotory Effect on Soybean. Sci. Rep. 2018, 8, 6650.

(20) Rossi, L.; Fedenia, L. N.; Sharifan, H.; Ma, X.; Lombardini, L. Effects of Foliar Application of Zinc Sulfate and Zinc Nanoparticles in Coffee (Coffea Arabica L.) Plants. Plant Physiol. Biochem. 2019, 135, $160-166$.

(21) Karny, A.; Zinger, A.; Kajal, A.; Shainsky-Roitman, J.; Schroeder, A. Therapeutic Nanoparticles Penetrate Leaves and Deliver Nutrients to Agricultural Crops. Sci. Rep. 2018, 8, 7589.

(22) Lv, J.; Christie, P.; Zhang, S. Uptake, Translocation, and Transformation of Metal-Based Nanoparticles in Plants: Recent Advances and Methodological Challenges. Environ. Sci.: Nano 2019, 6, 41-59.

(23) Giraldo, J. P.; Landry, M. P.; Faltermeier, S. M.; McNicholas, T. P.; Iverson, N. M.; Boghossian, A. A.; Reuel, N. F.; Hilmer, A. J.; Sen, F.; Brew, J. A.; et al. Plant Nanobionics Approach to Augment Photosynthesis and Biochemical Sensing. Nat. Mater. 2014, 13, 400408. 
(24) Choudhary, R. C.; Kumaraswamy, R. V.; Kumari, S.; Sharma, S. S.; Pal, A.; Raliya, R.; Biswas, P.; Saharan, V. Cu-Chitosan Nanoparticle Boost Defense Responses and Plant Growth in Maize

(Zea Mays L.). Sci. Rep. 2017, 7, 9754.

(25) Saharan, V.; Kumaraswamy, R. V.; Choudhary, R. C.; Kumari, S.; Pal, A.; Raliya, R.; Biswas, P. Cu-Chitosan Nanoparticle Mediated Sustainable Approach to Enhance Seedling Growth in Maize by Mobilizing Reserved Food. J. Agric. Food Chem. 2016, 64, 6148-6155.

(26) Pereira, A. E. S.; Sandoval-Herrera, I. E.; Zavala-Betancourt, S. A.; Oliveira, H. C.; Ledezma-Pérez, A. S.; Romero, J.; Fraceto, L. F. $\gamma$ Polyglutamic Acid/Chitosan Nanoparticles for the Plant Growth Regulator Gibberellic Acid: Characterization and Evaluation of Biological Activity. Carbohydr. Polym. 2017, 157, 1862-1873.

(27) Maruyama, C. R.; Guilger, M.; Pascoli, M.; Bileshy-José, N.; Abhilash, P. C.; Fraceto, L. F.; De Lima, R. Nanoparticles Based on Chitosan as Carriers for the Combined Herbicides Imazapic and Imazapyr. Sci. Rep. 2016, 6, 19768.

(28) Liang, W.; Yu, A.; Wang, G.; Zheng, F.; Jia, J.; Xu, H. ChitosanBased Nanoparticles of Avermectin to Control Pine Wood Nematodes. Int. J. Biol. Macromol. 2018, 112, 258-263.

(29) Liang, Y.; Fan, C.; Dong, H.; Zhang, W.; Tang, G.; Yang, J.; Jiang, N.; Cao, Y. Preparation of MSNs-Chitosan@Prochloraz Nanoparticles for Reducing Toxicity and Improving Release Properties of Prochloraz. ACS Sustainable Chem. Eng. 2018, 6, 1021110220.

(30) Sharma, A.; Sood, K.; Kaur, J.; Khatri, M. Agrochemical Loaded Biocompatible Chitosan Nanoparticles for Insect Pest Management. Biocatal. Agric. Biotechnol. 2019, 18, 101079.

(31) Parisi, C.; Vigani, M.; Rodríguez-Cerezo, E. Agricultural Nanotechnologies: What Are the Current Possibilities? Nano Today 2015, 10, 124-127.

(32) Yin, J.; Wang, Y.; Gilbertson, L. M. Opportunities to Advance Sustainable Design of Nano-Enabled Agriculture Identified through a Literature Review. Environ. Sci.: Nano 2018, 5, 11-26.

(33) Wang, P.; Lombi, E.; Zhao, F.-J.; Kopittke, P. M. Nanotechnology: A New Opportunity in Plant Sciences. Trends Plant Sci. 2016, 21, 699-712.

(34) Larignon, P.; Dubos, B. Fungi Associated with Esca Disease in Grapevine. Eur. J. Plant Pathol. 1997, 103, 147-157.

(35) Bertsch, C.; Ramírez-Suero, M.; Magnin-Robert, M.; Larignon, P.; Chong, J.; Abou-Mansour, E.; Spagnolo, A.; Clément, C.; Fontaine, F. Grapevine Trunk Diseases: Complex and Still Poorly Understood. Plant Pathol. 2013, 62, 243-265.

(36) Fungicide Resistance in Plant Pathogens; Ishii, H., William, D., Eds.; Springer: Tokyo, Japan, 2015.

(37) Balakshin, M.; Capanema, E. On the Quantification of Lignin Hydroxyl Groups with ${ }^{31} \mathrm{P}$ and ${ }^{13} \mathrm{C}$ NMR Spectroscopy. J. Wood Chem. Technol. 2015, 35, 220-237.

(38) Mather, B. D.; Viswanathan, K.; Miller, K. M.; Long, T. E. Michael Addition Reactions in Macromolecular Design for Emerging Technologies. Prog. Polym. Sci. 2006, 31, 487-531.

(39) Genest, A.; Portinha, D.; Fleury, E.; Ganachaud, F. The AzaMichael Reaction as an Alternative Strategy to Generate Advanced Silicon-Based (Macro)Molecules and Materials. Prog. Polym. Sci. 2017, 72, 61.

(40) Antonietti, M.; Landfester, K. Polyreactions in Miniemulsions. Prog. Polym. Sci. 2002, 27, 689-757.

(41) Bechthold, N.; Tiarks, F.; Willert, M.; Landfester, K.; Antonietti, M. Miniemulsion Polymerization: Applications and New Materials. Macromol. Symp. 2000, 151, 549-555.

(42) Tripathi, D. K.; Shweta; Singh, S.; Singh, S.; Pandey, R.; Singh, V. P.; Sharma, N. C.; Prasad, S. M.; Dubey, N. K.; Chauhan, D. K. An Overview on Manufactured Nanoparticles in Plants: Uptake, Translocation, Accumulation and Phytotoxicity. Plant Physiol. Biochem. 2017, 110, 2-12. 